Mediterránea Ser. Biol. (1990), n. 12. Pág. 89-96

\title{
ASPECTOS DE LA REPRODUCCIÓN DE LA TÓRTOLA COMÚN (STREPTOPELIA TURTUR, L.) EN MADRID
}

\author{
por \\ V. PEIRÓ(1)
}

\section{RESUMEN}

Este estudio ha sido realizado en Quijorna (Madrid) durante los años 1983 y 84 . Se trata de una zona con cultivos de cereales, sotos fluviales, encinares y olivares. La estación reproductora de la Tórtola Común se extiende, principalmente, desde la primera quincena de mayo a la segunda de agosto. El tamaño medio de puesta es de 1.98 huevos y el tamaño de pollada de 1.87 pollos. El éxito reproductor difiere claramente entre los dos años de estudio, siendo bajo en 1983. La pérdida de huevos es debida, principalmente, al abandono y/o depredación. La mortalidad de pollos se debe a la depredación. El periodo de caza de la Tórtola Común debería iniciarse en septiembre para evitar un efecto negativo sobre la reproducción.

PALABRAS CLAVE: Streptopelia turtur, Madrid (España), Estación reproductora, Puesta, Pollada, Éxito reproductor.

\section{SUMMARY}

This study has been carried out in the 1983-84 breeding seasons in Quijorna (Madrid). This area is a cereal producing district with fluvial groves, holm-oak woods and olive groves. The breeding seasons extends from the first fortnight of May to the second fortnight of August. The mean clutch size was 1.98 , while the mean number of nestlings was 1.87 . The breeding success differs clearly between two years, beings low in 1983. The losses of eggs are mainly due to desertion and/or predation. Nestlings' mortality is due to predation. The hunting season of the turtle dove should start in September, so as to avoid the effect of hunting on breeding.

KEY WORDS: Streptopelia turtur, Madrid (Spain), Breeding season, Clutch, Nestling, Breeding success.

(1) Cátedra de Vertebrados. Facultad Biología. Univ. Complutense Madrid. 28040-Madrid. Dirección actual: Dept. Ciencias Ambientales y R.N.-Biología. Facultad Ciencias. Uni. Alicante. Apt. ${ }^{\circ}$ 99. 03080-Alicante. 


\section{INTRODUCCIÓN}

La información actual sobre la biología reproductora de la Tórtola Común (Streptopelia turtur) es escasa y basada en estudios muy puntuales (HEER, 1963; BRAAKSMA, 1968; ERARD, 1969; WALLIS, 1970). La estación y el éxito de la reproducción han sido estudiados con profundidad en Gran Bretaña (MURTON, 1968). En España, la información sobre la evolución cuantitativa de dichos aspectos es prácticamente inexistente. Pero, sobre todo, destaca la escasez de conocimientos sobre la duración de la estación reproductora, y por tanto, la imposibilidad de disponer de datos que permitan fijar unos períodos hábiles de caza, que no influyan negativamente sobre la reproducción de la especie.

Los objetivos concretos del presente artículo son: a) determinar cualitativa y cuantitativamente la duración de la estación reproductora; b) aportar nuevos datos al conocimiento actual sobre la puesta y la pollada; c) cuantificar el éxịto reproductor y la mortalidad que influyen sobre los huevos y los pollos.

\section{ÁREA DE ESTUDIO}

El área de estudio está situada en el cuadrante SW (a $40 \mathrm{Km}$. de Madrid, capital) de la provincia de Madrid $\left(40^{\circ} 25^{\prime} \mathrm{N} ; 04^{\circ} 03^{\prime} \mathrm{W}\right.$ ), en el coto privado M-10125. El relieve está constituido por un conjunto de cerros redondeados, separados por un sistema hidrológico formado por el arroyo de Quijorna y el arroyo de la Candalosa. El clima es mediterráneo semiárido (IZCO, 1984), con ombroclima seco $(400-500 \mathrm{~mm}$.).

La vegetación está constituida por cultivos de trigo (Triticum spp.) y olivo (Olea europaea), formaciones de encina (Quercus rotundifolia) y sotos con olmo (Ulmus spp.), chopo (Populus spp.), sauce (Salix spp.), quejigo (Quercus faginea), majuelo (Crataegus monogyna) y zarza (Rubus spp.).

\section{MATERIAL Y MÉTODOS}

El estudio se llevó a cabo durante los años 1983 y 84 . La búsqueda de nidos se realizó con visitas semanales (1983) y quincenales (1984); pero cuando los nidos tenían pollos, se visitaban cada tres o cuatro días. El método de búsqueda seguido fue el de sucesivas idas y venidas en paralelo. En los dos años se encontraron un total de 132 nidos. Los datos meteorológicos fueron obtenidos del observatorio Madrid-Retiro.

\section{RESULTADOS}

\section{La estación reproductora, la puesta y la pollada}

La figura 1 muestra la duración de la estación reproductora y su evolución cuantitativa, con respecto al número de nidos, en cada uno de los dos años de estudio (1983-84). 

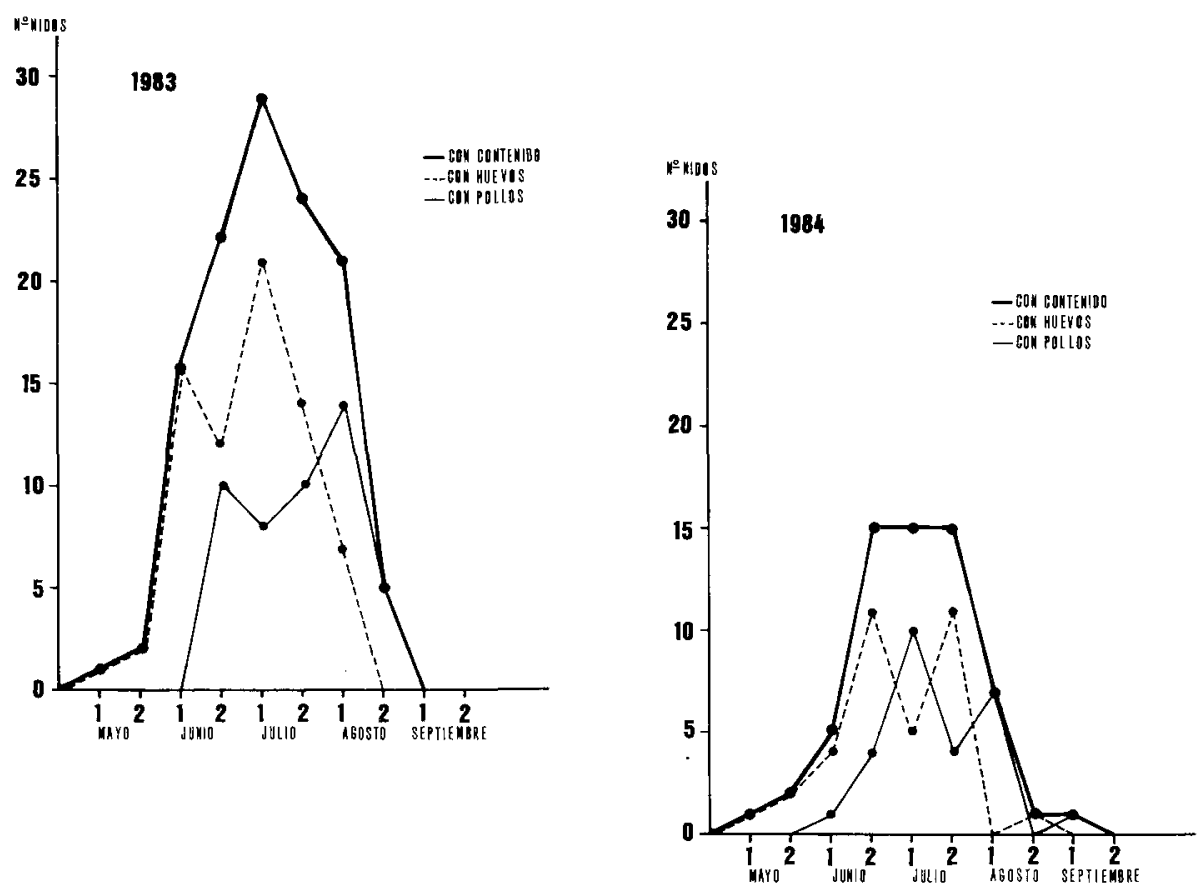

Figura 1.- Estación reproductora en los años 1983 y 1984, basada en el número de nidos con contenido, con huevos y con pollos en cada quincena. (Breeding season in the years 1983 y 1984, based on the number of occupied nests, with eggs and with nestlings, in fortnightly periods).

La reproducción empezó, en ambos años, en la primera quincena de mayo y finalizó en la segunda quincena de agosto, si bien en 1984 existe un nido tardío que finalizó la crianza de los pollos en la primera quincena de septiembre. La duración de la estación reproductora fue de 98 días en 1983 (13-V al 19-VIII) y 100 días en 1984 (15-V al 23-VIII); aunque si se incluye el nido tardío de este segundo año, la duración será de 118 días (hasta el 10-IX).

La figura 2 presenta la evolución mensual de la temperatura mínima media y de los días de precipitación, considerados como dos factores potencialmente condicionantes de la estación reproductora. En esta figura se observa que la temperatura mínima media mensual toma valores similares en ambos años, por lo que no se podría deducir, con dichos datos, que la temperatura influya en la diferente evolución de la estación reproductora (sobre la influencia de la precipitación, ver discusión).

La Tórtola Común presenta, en la zona de estudio, un tamaño medio de puesta de 1.98 huevos $(\mathrm{s}=0.14 ; \mathrm{n}=107)$ y no aparecen diferencias interanuales significativas $\left(\chi^{2}=1.39\right.$; n.s.). El tamaño medio de pollada ha sido de 1.87 $(\mathrm{s}=0.34 ; \mathrm{n}=70)$, no existiendo diferencias entre años $\left(\chi^{2}=0.23 ; \mathrm{n} . \mathrm{s}.\right)$. 

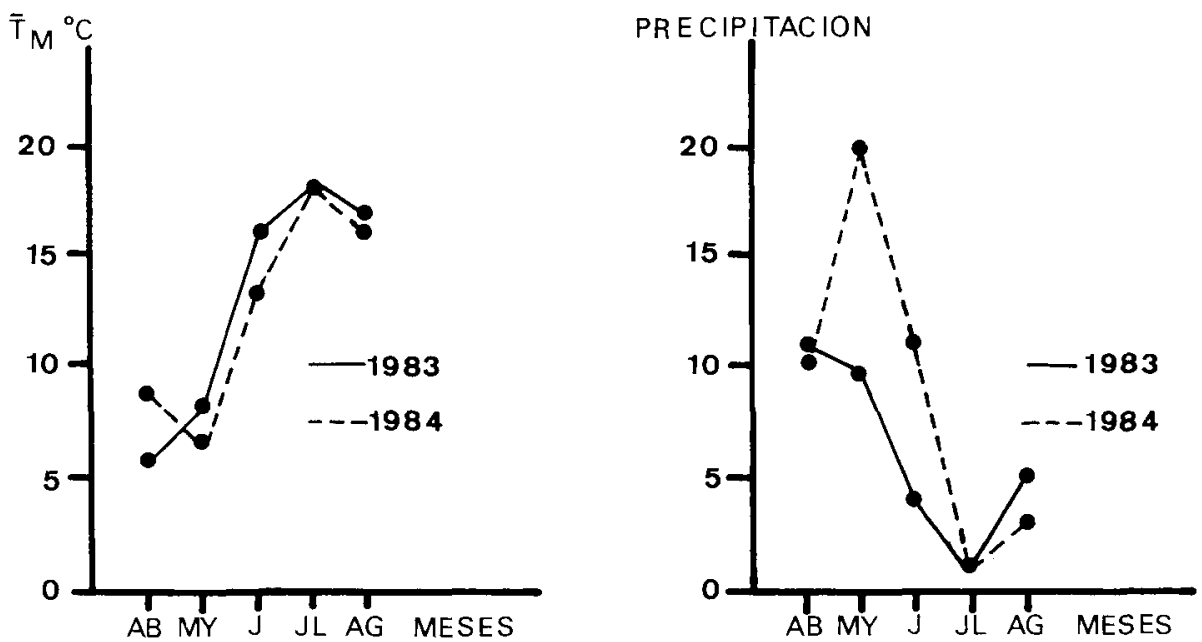

Figura 2.- Temperatura mínima media y días de precipitación mensuales en cada uno de los dos años de estudio. Datos del observatorio más próximo (Madrid-Retiro). (Monthly minimal mean temperature and rainfall days for both study years. Data from the nearest observatory, Madrid-Retiro).

\section{Éxito y mortalidad reproductores}

La tabla I presenta el éxito de la reproducción como el porcentaje de nidos en los que eclosionan los huevos y son criados los pollos. Las diferencias interanuales son significativas en el éxito de eclosión $\left(\chi^{2}=6.07 ; \mathrm{p}<0.05\right)$ y en el éxito reproductor $\left(\chi^{2}=4.66 ; p<0.05\right)$, mientras que no existen diferencias en el éxito de pollos $\left(\chi^{2}=0.44\right.$, n.s.). En dicha tabla se observa que el éxito reproductor es mucho menor en 1983 y presenta un valor bajo. Además, en 1984, el éxito de eclosión es mayor que el éxito de pollos; mientras, en 1983, el primer éxito es menor que el segundo.

En 1983, el éxito de pollos respecto al mes en que nacen éstos (tabla II) toma sus valores máximo y mínimo en junio y julio, respectivamente, mientras, en 1984, ocurre de forma inversa. Las diferencias porcentuales entre años son significativas $\left(\chi^{2}=9.21 ; \mathrm{p}<0.01\right)$.

Las pérdidas en estado de huevos se deben, principalmente, al caso conjunto de abandono y/o depredación. La separación total de ambas causas no ha podido realizarse, debido a la imposibilidad de determinar, en nidos con cascarones rotos o en nidos donde los huevos han desaparecido, si se trata de verdaderas depredaciones o si previamente ha existido abandono.

La pérdida de un solo pollo aparece en 8 nidos $(8 \%)$, mientras que la pérdida de los dos pollos se produjo en 20 nidos $(20 \%)$. Conjuntamente, dichas 
TABLA I

Éxitos de eclosión, pollos y reproductor en cada uno de los dos años de estudio (Hatching success, nestling success and breeding success for both study years)

\begin{tabular}{|c|c|c|c|c|}
\hline AÑO & $\begin{array}{l}N .{ }^{\circ} \text { de nidos } \\
(N . \text { of nests) }\end{array}$ & $\begin{array}{l}\text { \% nidos con huevos } \\
\text { eclosionados }(1) \\
\text { (\% nests with } \\
\text { hatched eggs) }\end{array}$ & $\begin{array}{l}\% \text { nidos con pollos } \\
\text { criados respecto al de } \\
\text { huevos eclosionados }(2) \\
\text { (\% nests with fledged } \\
\text { youngs from the nests } \\
\text { with hatched eggs) }\end{array}$ & $\begin{array}{c}\% \text { nidos con pollos } \\
\text { criados respecto al de } \\
\text { huevos puestos ( } 3 \text { ) } \\
\text { (\% nests with fledged } \\
\text { youngs from the nests } \\
\text { with laid eggs) }\end{array}$ \\
\hline 1983 & 64 & $55 \%$ & $66 \%$ & $36 \%$ \\
\hline 1984 & 38 & $79 \%$ & $73 \%$ & $58 \%$ \\
\hline
\end{tabular}

(1) Éxito de eclosión (hatching success). (2) Éxito de pollos (nestling success).

(3) Éxito reproductor (breeding success).

\section{TABLA II}

Éxito de pollos, en relación al mes en que nacen, en cada uno de los dos años de estudio. (Nestling success in relation to hatching month for both study years)

\begin{tabular}{lcccc} 
& \multicolumn{2}{c}{$\begin{array}{c}N^{\circ}{ }^{\circ} \text { de pollos nacidos } \\
\text { (N. of hatched young) }\end{array}$} & \multicolumn{2}{c}{$\begin{array}{c}\% \text { pollos criados } \\
\text { (\%) fledged young) }\end{array}$} \\
\hline JUNIO & 1983 & 1984 & 1983 & 1984 \\
JULIO & 16 & 8 & $100 \%$ & $50 \%$ \\
AGOSTO & 27 & 22 & $41 \%$ & $86 \%$ \\
\hline
\end{tabular}

pérdidas no difieren entre los dos años de estudio $\left(\chi^{2}=0.22\right.$; n.s. $)$. La mortalidad en estado de pollos es debida a la depredación. Los depredadores cuya acción ha sido constatada son la Culebra de Escalera (Elaphe scalaris), una rapaz diurna, córvidos (Pica pica o Cyanopica cyana) y el hombre (hurto de pollos).

\section{DISCUSIÓN}

La estación reproductora de la zona de estudio concuerda con la bibliografía existente en otros países europeos (JOURDAIN, 1965; DEMENTIEV, 1968; MURTON, 1968; CRAMP, 1985). Sin embargo, no se puede realizar 
ninguna comparación con otras zonas españolas, puesto que la duración de la estación reproductora de esta especie es insuficientemente conocida en la Península Ibérica.

Respecto a los factores condicionantes de la reproducción, la precipitación no influye en el inicio de ésta, puesto que con una mayor precipitación en mayo de 1984 (ver fig. 2), los gráficos de la estación reproductora de ambos años (ver fig. 1) presentan un trazado idéntico para este mes. La precipitación influye indirectamente en la evolución cuantitativa de los nidos durante la estación reproductora. Esta influencia vendría determinada por la mayor pluviometría de mayo y junio de 1984; lo que, según estimaciones cualitativas, produjo una fuerte proliferación de herbáceas silvestres en mayo, con el consiguiente aumento de semillas disponibles entre la segunda quincena de junio y la primera de julio. Esta mayor disponibilidad de alimento explicaría que el máximo de nidos con pollos de 1984 se alcance en este período. Sin embargo, en 1983, el máximo de nidos con pollos se alcanza en la primera quincena de agosto, un mes después que el de huevos, lo que indicaría que algún otro factor debe de actuar sobre la estación reproductora de este año.

En 1984, la mayor disponibilidad de semillas herbáceas (apreciaciones cualitativas) también debió de influir en el mayor éxito de este año. Si a ésta se le une que los cereales ya han madurado a principios de julio, se explica que el éxito de pollos respecto al mes de eclosión tenga lugar en julio de este año. En 1983 no existe tal concordancia, por lo que el éxito debe de estar determinado por un factor diferente al alimentario. En Gran Bretaña (MURTON, 1968), la especie también tiene un éxito mayor en julio, que es cuando encuentra mayor cantidad de semillas silvestres y dispone del suplemento de los cereales.

Por último, si se compara la duración de la estación reproductora (como mínimo, hasta la segunda quincena de agosto) con el período hábil de caza de la Tórtola Común en España (primera quincena de agosto hasta la segunda de septiembre), se deduce que la actividad cinegética se inicia cuando todavía no ha finalizado la reproducción de esta especie, provocando la pérdida de crianzas al ser abatidos los progenitores. Esta causa de pérdida ha sido constatada por observaciones del autor en Almenara (Castellón) y por la existencia de «leche de paloma» en buches de tórtolas abatidas durante la segunda quincena de agosto en Cañamero, Cáceres, (datos del autor).

Aunque se debería disponer de datos de mayor número de provincias, sería conveniente no permitir la apertura de la caza de la tórtola común hasta los primeros días de septiembre. De este modo, la influencia negativa de la actividad cinegética sobre la reproducción de esta especie será mínima o nula.

\section{AGRADECIMIENTOS}

Deseo agradecer al Dr. D. Francisco Bernis por su dirección; a D. Isidoro Murta Neves, por sus comunicaciones; a L. M. Carrascal y E. Seva, así como a otros revisores que con su crítica contribuyeron a mejorar el manuscrito original. 
BRAAKSMA, S. (1968). Vroeg broedsel van der Tortelduif (Streptopelia turtur). Limosa 41: 115. CRAMP, S. (1985). The Turtle Dove. Handbook of the birds of Europe, the Middle East and North Africa. Vol. IV, pp. 353-363. Oxford University Press. London.

DEMENTIEV, G. P. (1968). Turtle Dove. Birds of the Soviet Union. Vol. II, pp. 47-53. Israel Program for Scientific Traslations, Keter Press. Jerusalem.

ERARD, C. (1969). Durrée d'incubation anormale chez Streptopelia turtur, L'Oiseau et R.F.O. 39: 268-269.

HEER, H. (1963). Notizen zur fortflanzungsbiologie der Turkentaube. Jh. Ver. Vartel. Naturk. Wurtt. 125: 276-278.

IZCO, J. (1984). Madrid Verde. Instituto de Estudios Agrarios, Pesqueros y Alimentarios. Madrid. JOURDAIN, F. C. R. (1965). The Turtle Dove: Breeding. Handbook of the British Birds. T. IV, pp. 142. Ed. Witherby's.

MURTON, R. K. (1968). Breeding, migration and survival of the Turtle Dove. British Birds 61: 193-212.

WALLIS, G. (1970). Unusual nest-side for Turtle Dove. Naturalist Hull. 912: 5. 